\title{
ESTIMATION THEORETIC FRAMEWORK FOR COMPARING POLARIZATION BASED, CONTINUOUS-WAVE DIRECT IMAGING SCHEMES
}

\author{
Umesh R S, Ramakrishnan A G, Srikanth R \\ Indian Institute of Science \\ Electrical Engineering Department \\ Bangalore, India - 560012
}

\author{
Hema Ramachandran \\ Raman Research Institute \\ Optics Laboratory \\ Bangalore, India - 560012
}

\begin{abstract}
We report a maiden study and comparison of two important classes of polarization based continuous-wave optical imaging schemes for imaging through scattering media, namely, the polarization difference Imaging (PDI) and the Polarization Modulation Imaging (PMI). We cast the problem in an estimation theoretic framework and base the comparison on two visualization parameters, the polarization magnitude and the degree of polarization. We show that PDI is superior in estimating these two parameters in active imaging. However, we show that PMI is suitable for passive imaging and that the PDI is a specific implementation of PMI.
\end{abstract}

\section{INTRODUCTION}

In direct optical imaging, the sole aim is to use only unscattered light for imaging [1]. The criteria for rejecting and accepting different parts of the radiation as scattered and unscattered varies, and gives rise to different imaging schemes. In this study, we consider only continuous-wave, direct imaging schemes which use linear polarization of the received radiation to discriminate the unscattered from the scattered part. From this study, parallcls can be drawn to the case of circular polarization.

Many schemes subtract sets of co-polarized and crosspolarized images or their scaled versions, to obtain the image corresponding to the unscattered component of light $[2,3]$ and hence belong to the class of PDI. There are other schemes which use polarization modulation followed by sinusoidal estimation $[4,5]$ and achieve the same goal. These belong to the category of PMI. These schemes are used to measure either the intensity of polarized light (Polarization intensity imaging - PII) $[4,5]$, or the degree of linear polarization (DOLP [6]) of the received radiation $[2,3]$. PDI has been shown to be capable of measuring these parameters $[2,3]$. However, PMI has been used only for PII, though, as we show, it can also be used for measuring DOLP. Besides, PDI is useful for both active and passive tmaging, whereas PMI described in $[4,5]$ is useful only for active imaging.

However, PMI can be made suitable for both active and passive imaging by keeping the plane of polarization of the incident light fixed and allowing the analyzer to rotare. Then, we can observe that PDI becomes a particular case of this modified PMI scheme if images are captured at angular displacements of $\pi / 2$ of the analyzer. The frequency of the resulting sinusoid due to unscattered light will be half the sampling rate, i.e., only two points of the sinusoid will be sampled per period. If one of the sampled points (images) is chosen to be at the maximum of the sinusoid (the co-polarized image), the next sample will naturally be that of the minimum of the sinusoid (the cross-polarized image). Thus, we can get the PDI data by properly choosing the sampling point in the modified PMI scheme. Hence, PDI is a particular case of the more versatile PMI. Henceforth, all references to PMI essentially refer to this modified PMI scheme.

We now build a framework to study these imaging schemes, wherein we treat them as different estimators of polarization intensity and DOLP.

\section{SIGNAL MODELING}

As we observed, it is sufficient to model the data obtained by PMI, since we can easily obtain PDI data from the same. We model the observed data (images obtained), by using the Stokes vectors (SV) $[6,7,8]$. In general, the SV recorded at a pixel location of a

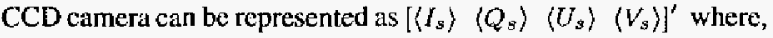
(i) represents time averaging. Though, due to finite detector area, there is spatial averaging also, we are more interested in time averaging, since the areas involved are small and the dominant factor affecting the recorded $S V$ turns out to be time. We assume that the integration times are usually large compared to the coherence time of the sources [9], and hence, by virtuc of the central-limit theorem, the time averaged $S V$ recorded during different sub-intervals can be assumed to be Gaussian iid random variables [9].

Though this assumption seems reasonable for most of the data collected, in some cases, data revealed an underlying coloured noise process, which could be modeled by an ARI process. Hence, in our model, we do not assume the noise to be white. By Malu's law [8], if light with input SV $S_{i n}=\left(I_{i} Q_{i} U_{i} V_{i}\right)^{T}$ is incident on a linear polarizer, the intensity of the output $S V$ is related to the input parameters by the relation

$$
I_{o}=\frac{1}{2}\left(I_{i}+Q_{i} \cos 2 \theta+U_{i} \sin 2 \theta\right)
$$

Thus, the intensity recorded at an arbitrary pixel location at the $n^{\text {th }}$ step of acquisition of PMI can be represented as

$I_{\mathrm{r}}(n)=\frac{1}{2}\left(I_{s}+Q_{s} \cos 2 \theta_{n}+U_{s} \sin 2 \theta_{n}\right)+v(n), \quad n=0, \cdots, N-1$

where $\theta_{n}$ is the angle made by the analyzer with respect to the horizontal at the $n^{\text {th }}$ step and $v(n)$ in general, represents a coloured noise process. Assuming that the rotating polaroid takes $M$ steps to complete exactly one rotation, $\theta_{n}$ increments by $\frac{2 \pi}{M}{ }^{c}$ with each step of the polaroid. Suppose we start grabbing images with an arbitrary orientation $\phi$ of the rotating polaroid, the intensity recorded at the $n^{\text {th }}$ step can be represented as

$$
I_{\Gamma}(n)=\frac{1}{2}\left(I_{s}+\sqrt{Q_{s}^{2}+U_{s}^{2}} \sin \left(\frac{4 \pi n}{M}+2 \phi+\alpha\right)\right)+v(n)
$$


where, $\alpha=\arctan \left(Q_{s} / U_{s}\right)$. We observe from (1) that the component of the intensity that is independent of the orientation of the analyzer is $I_{s} / 2$, which corresponds to the diffuse part of the scattered light. The amplitude of the sinusoidal part, i.e., $\sqrt{Q_{s}^{2}+U_{s}^{2}} / 2$ corresponds to the unscattered component which would be polarized, and hence, is a measure of the magnitude of the polarized light in the received radiation. We denote the former by $I_{\text {scat }}$ and the latter by $I_{b a i}$. Using this representation,

$I_{r}(n)=I_{\text {scat }}+I_{\text {bal }} \sin \left(\frac{4 \pi n}{M}+\beta\right)+v(n) \quad n=0, \cdots, N-1$ (2) where we have replaced the term $2 \phi+\alpha$ by a single variable, $\beta$. The discrete frequency $f$ of $I_{b a l}$ is given by $f=2 / M$. In all our comparisons of the imaging schemes, we assume that there are $N$ images available for analysis. i.e., in case of PDI, there would be $N / 2$ images each, corresponding to the co-polarized and cross-polarized data. In PMI schemes, there would be $N$ images constituting a time series at every pixel location, which would be analyzed for estimating the sinusoidal component. We further assume that $N$ is an integral multiple of $M$ (however, this condition is not strict for $N$ large).

The comparison of the analysis schemes needs the knowledge of the noise statistics at each pixel location, which is seldom known a priori. Still, we assume that the noise characteristics are known and analyze the various schemes, since we get an idea of the performance of the various estimators given a particular noise condttion. We do not explicitly estimate the noise variance terms, since the quantity of interest to us is the unscattered component of light and noise is a nuisance parameter. We now compare the PDI and PMI, based on this model.

\section{POLARIZATION INTENSITY IMAGING - COMPARISON OF PDI AND PMI SCHEMES}

\subsection{The case of white noise}

\subsubsection{The PDI estimator}

We denote the co-polarized intensity recorded in a general PDI scheme by $I_{\|}$and the cross-polarized intensity by $I_{\perp}$. Since the plane of polarization of the incident light is not known in general, these intensities need not correspond to the exact co-polanization and cross-polarization locations. We assume that the intensity recorded with the analyzer at an angle $\phi$ with respect to the horizontal as $I_{\|}$. Thus the recorded co-polarized and cross-polarized intensities will be (from (2))

$$
\begin{gathered}
I_{\|}(n)=I_{s c a t}+I_{b a l} \sin (\beta)+u(n) \\
I_{\perp}(n)=I_{s c a t}+I_{b a l} \sin (\beta+\pi)+w^{\prime}(n)
\end{gathered}
$$

where $w(n)$ and $w^{\prime}(n)$ are zero mean iid Gaussian random variables and $\beta=(2 \phi+\alpha)$. Using PDl, the estimate of the unscattered component in the recorded data can be obtained as

$$
\hat{I}_{b a l, P D I}=\frac{1}{N} \sum_{n=1}^{\frac{N}{2}}\left(I_{\|}(n)-I_{\perp}(n)\right)
$$

In some implementations of PDI, the scaling factor is an arbitrary constant $[10,2]$. However, the conect theoretical estimate of the unscattered component is given only by (5). Now, by substituting the expressions for $I_{1}$ and $I_{\perp}$ from (3) and (4) into (5), and by simplifying the resulting expression, we obtain,

$$
\hat{I}_{b a l, P D I}=I_{b a l} \sin \beta+w^{*}(n)
$$

where $w^{*}(n)$ is a zero-mean, Gaussian iid noise, with variance $\sigma^{2} / N$, where $\sigma^{2}$ is the variance of $w(n)$ and $w^{\prime}(n)$. We can observe from (6) that

$$
E\left\{\tilde{I}_{b a l, P D I}\right\}=I_{b a l} \sin \beta
$$

$$
\operatorname{var}\left\{\hat{I}_{b a l, P D I}\right\}=\sigma^{2} / N
$$

Clearly, the estimate $\bar{I}_{b a t, F D I}$ is biased, since the estimated value depends upon $\beta$. This is a big disadvantage of PDI. Only when $\beta=\pi / 2$, we get the true estimate of the unscattered component. The estimator has a variance of $\sigma^{2} / N$, irrespective of the value of $\beta$. Next, we analyze the performance of the PMI polarization intensity estimator.

\subsubsection{The PMI estimator}

Suppose $N$ data values from PMI are available for analysis. It can be observed from (2) that the $\frac{2 N}{M I}^{\text {th }}$ component of the N-point DFT would contain the information of the sinusoid. The estimate of $I_{b a l}$, is given by

$$
\hat{I}_{b a l, P M I}=\frac{2}{N}\left|\mathbf{I}_{\mathbf{r}}\left(\frac{2 N}{M}\right)\right|
$$

Here again, some PMI schemes use a different scaling factor [4]. The analysis of the bias and variance of this estimator is quite formidable, and hence we have resorted to numerical simulations. We compare the results of the simulation with that of the PDI intensity estimator in a later section. Next, we analyze as to what best can be done to estimate $I_{b a i}$, from the basic principles of estimation theory.

The best estimator, with the optimality criterion being the minimum variance of the estimated quantity, is known to be the minimum variance, unbiased (MVU) estimator [11]. To determine whether an MVU estimator exists, we have to see if some estimator satisfies the Cramer-Rao Lower bound (CRLB). Here, the quantity that we wish to estimate is $I_{b a t}$ of (2). However, it can be easily found that an MVU estimator does not exist for the estimation of $I_{b a l}$ using PML. Hence, we resort to estimate the Maximum Likelihood Estimator (MLE) of $I_{b a l}$. since MLE is known to be asymptotically efticient and optimal. Moreover, if an efficient estimator exists, it will be achieved by the MLE [11].

The problem on hand is similar to estimating the amplitude of a single sinusoid, except for the constant term $I_{\text {scot }}$. We explore whether we can extend the analysis of the case of a single sinusoid, detailed in [11] to that of ours. By modifying (2) as given below, it is clear that the data can be modeled linearly as

$$
\begin{gathered}
I_{r}(n)=I_{\text {scat }}+I_{b a l} \cos \beta \sin \left(\frac{4 \pi n}{M}\right)+I_{b a l} \sin \beta \cos \left(\frac{4 \pi n}{M}\right) \\
+w(n) \quad n=0, \cdots, N-1
\end{gathered}
$$

With this modification, we can express the above equation as

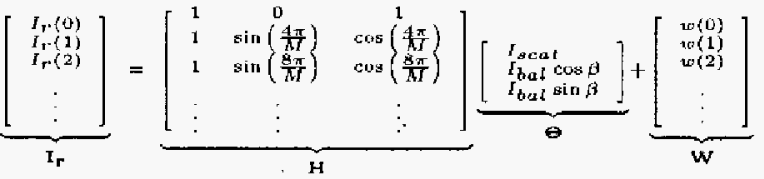

or, with matrix notation as

$$
I_{\mathbf{r}}=\mathbf{H} \Theta+\mathbf{W}
$$

If we can estimate $I_{b a l} \cos \beta$ and $I_{b a l} \sin \beta$, we can estimate $I_{b a l}$. It has been proved that, for data represented as a linear form as above, the MLE of $\Theta$ is [11]

$$
\hat{\Theta}=\left(\mathbf{H}^{\mathbf{T}} \mathbf{C}^{-1} \mathbf{H}\right)^{-1} \mathbf{H}^{\mathbf{T}} \mathbf{C}^{-1} \mathbf{X}
$$

$\hat{\Theta}$ is also an efficient estimator, in that it attains the CRLB and hence is the MVU estimator. Thus, we have efficient estimators for 
$I_{b a l} \cos \beta$ and $I_{b a l} \sin \beta$ and thus the MLE estimator for $I_{b a l}$ [11] is given by

$$
\hat{I}_{b a i, M L E}=+\sqrt{\left(I_{b a l} \cos \beta\right)^{2}+\left(I_{b a l} \sin \beta\right)^{2}}
$$

Although $I_{b a l}$ is biased if data points are few; it is asymptotically unbiased [12]. Moreover. since the estimate is obtained by a nonlinear transformation of the MVU estimates, the estimator cannot be efficient [11]. The MLE estimate $\hat{\Theta}$ for various components tums out to be

$$
\begin{gathered}
\hat{I}_{s c a t, M V U}=\frac{1}{N} \sum_{n=0}^{N-1} I_{r}(n) \\
\hat{I}_{b a t} \cos \beta_{M V U}=\frac{2}{N} \sum_{n=0}^{N-1} I_{r}(n) \sin \left(\frac{4 \pi n}{M}\right) \\
\hat{I}_{b a l} \sin \beta_{M V U}=\frac{2}{N} \sum_{n=0}^{N-1} I_{r}(n) \cos \left(\frac{4 \pi n}{M}\right) .
\end{gathered}
$$

and

$$
\begin{gathered}
\operatorname{var}\left\{\hat{I}_{\text {scat }, M V U}\right\}=\frac{\sigma^{2}}{N} \\
\operatorname{var}\left\{\hat{I}_{b a l} \cos \beta_{M V U}\right\}=\operatorname{var}\left\{\hat{I}_{b a l} \sin \beta_{M V U}\right\}=\frac{2 \sigma^{2}}{N}
\end{gathered}
$$

From (11) and (12), it is clear that the right hand side of (9) is the same as that of (7). Hence, we arrive at the important result that the MLE estimate of $I_{b a l}$ can be obtained by PMI. It is worth observing that if $\beta=\frac{\pi}{2}$, we can obtain the MVU estimates of $I_{\text {bol }}$. Hence, if we know the exact orientation of the plane of polarization of the source, we car obtain the MVU estimates of $I_{b a l}$ by PMI. We end our search for better estimators of $I_{b a l}$ here, since the MLE estimator almost always does the best job, when the MVU estimator does not exist.

The exact phase relations can usually be known in the case of active imaging. Hence, for active imaging, PDI schemes are more useful than PMI. For passive imaging, PMI seems to be more suitable. However, there are applications where the parameter of interest is not the cxact value of the sinusoidal amplitude, but its relative value across the scene. Since PDI gives uniformly scaled values of the sinusoidal amplitude across the scene, it may be better to use PDI in such circumstances, since its variance is lower than that of PMI. With this, we conclude our analysis of the estimators for $I_{b a t}$ in white noise.

\subsection{The case of coloured noise}

The theoretical analysis of the estimators in coloured noise is a formidable cusk and hence we have resorted to Monte-Carlo simulations to compare the estimators. However, a few important observations can be made before proceeding to the results.

Due to the same reasons as in the ease of white noise, it has been found that we cannot obtain an MVU estimate of $I_{b a l}$ by PMI, and we have to be satisfied with the MLE estimate given by (8). However, what makes the coloured noise case different is, the noise covariance matrix $\mathbf{C}$ in this case will not be $\sigma^{2} \mathbf{I}$, and hence, the estimates depend explicitly on the covariance matrix terms. On the other hand, if the noise is white, we can obtain the MLE estimates of the amplitude, without having to know the noise variance per se. Usually, we do not get the noise samples alone since the data contains signal plus noise infonmation. This makes the estimation of the noise covariance matrix difficult. Hence, numerous estimators have been destgned to tackle this problem [12]. Among the matched-filter based estimation techniques mentioned in [12],

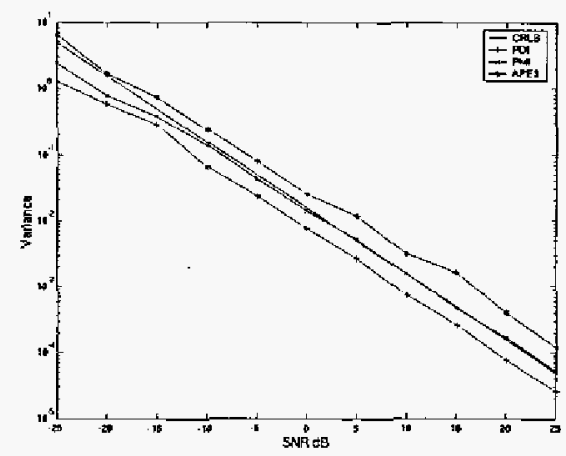

Fig. 1. Variance of PII estimators in white noise.

for our application, the Amplitude and Phase Estimator for Sinusoids (APES) algorithm scems to be the best suited, since we need to estimate the amplitude of one sinusoid only. We now compare the estimation results obtained from the PDI, PMI and the APES estimators, on the data generated using Monte-Carlo simulations.

\subsection{Results from Monte-Carlo simulations}

Simulations to test the PMI, MLE and the APES estimators used 200 realizations of 64 data points gencrated according to (2), with the signal frequency $f$ assumed to be 0.125 . For testing the PDI estimator, we used 32 data points each, with $\beta=\pi / 2$ and $3 \pi / 2$, corresponding to the co-polarized and the cross-polarized data, respectively. The values of $I_{s c a t}$ and $I_{b a t}$ are chosen to be 4 and 1 , respectively, so that the DOLP corresponds to 0.25. As we will observe, the performance of the estimators docs not depend on the values of $I_{s c a t}$ or $I_{b a l}$, but on the noise characteristics. Hence, even if we choose any other value of $I_{s c a t}$ and $I_{b a l}$, the bias and variance of the estimators will remain the same.

Various characteristics of the estimators were studied by varying the SNR through the variance of the iid Gaussian random variable governing the noise process [12]. For deciding the range of SNR values to be considered, we calculated the average SNR of a $10 \times 20$ region of eight data sets that we obtained experimentally. Such an analysis showed the actual SNR to vary from around $14 \mathrm{~dB}$ to $9 \mathrm{~dB}$. Hence, we have considered the SNR range from $-25 \mathrm{~dB}$ to $+25 \mathrm{~dB}$ for our analysis. Though we found that the noise process in the actual data could be adequately represented by an AR1 process, we have tested the algorithms with AR2 noise process too, so that the performance of the estimators in unknown noise conditions could be better understood.

For studying the performance of the estimators under ARI noise, we chose the AR coefficient $a$, to be 0.50 , in all the simulations. To study the performance of the estimators in AR2 noise, we chose the AR coefficients to be $a_{1}=0.50$ and $a_{2}=-0.125$, respectively. The pole frequency for this choice of AR coetficients corresponds to a discrete frequency of 0.125 , and coincides with the frequency of the sinusoid, thus creating a relatively difficult situation to estimate the sinusoidal amplitude. The performance of all the estimators were similar in AR1 and AR2 noise conditions. Hence, we do not report our observations on the performance of the estimators in AR2 noise.

Figs 1 and 2 show the performance of the various estimators 


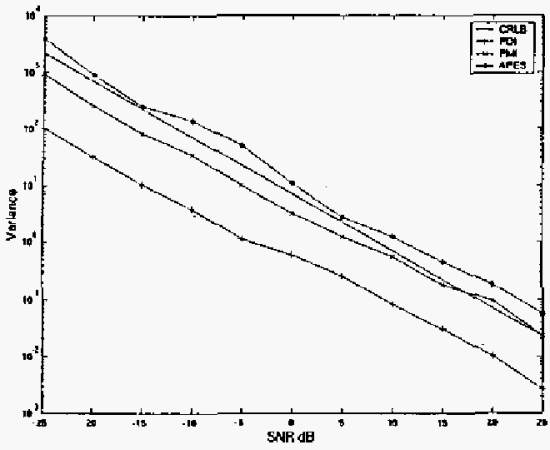

Fig. 2. Variance of PII estimators in AR 1 noise.

in white and coloured noise, respectively. We can observe that the PDI and PMI perform consistently better than the APES estimator. Though the PMI and MLE perform equally well, the high cornputational cost of the MLE favours the use of PMI. PDI exhibits lower bias and variance (at $\beta=\pi / 2$ ), endorsing our theoretical observations.

\section{DEGREE OF POLARIZATION IMAGING - COMPARISON OF PDI AND PMI SCHEMES}

An imaging scheme that uses DOLP as the visualization parameter is reported in [2], where, it is defined as

$$
D O L P=\frac{I_{\|}-I_{\perp}}{I_{\|}+I_{\perp}}
$$

$I_{\| \mid}$and $I_{\perp}$ refer to the co-polarized and cross-polarized intensities. By substituting $\beta=\pi / 2$ into ( 3 ) and (4), we obtain

$$
D O L P=\frac{I_{b a l}}{I_{s c a t}}=\frac{\sqrt{Q_{s}^{2}+U_{s}^{2}}}{I_{s}}
$$

Though DOLP has been explicitly defined for the PDI schemes, there is no such mention of a measure of DOLP in the PMI schemes given in $[4,5]$. It can be observed that, in PMI, the DOLP information can be obtained as the ratio of the amplitude of the sinusoidal component to the DC component. Thus, we observe that both the PDI and the PMI (and also, the APES) are capable of estimating polarization intensity and DOLP.

\subsubsection{The PDI estimators of DOLP}

A general PDI DOLP estimator can be mathematically expressed as,

$$
\widehat{D O L} P_{P D I}=\frac{1}{K} \sum_{k=1}^{K}\left(\frac{\sum_{n=0}^{\frac{N}{2 k}-1}\left(I_{\|}(n)-I_{\perp}(n)\right)}{\sum_{n=0}^{\frac{N}{2 K}-1}\left(I_{\|}(n)+I_{\perp}(n)\right)}\right)
$$

where, $I_{\|}(n)$ and $I_{\perp}(n)$ are given by (3) and (4). Here, we have assumed $N$ to be even, for mathematical convenience, though this is not a necessary condition. Two estimators which represent the extreme cases of the above general estimator are

$$
\widehat{D O L} P_{P D I 1}=\frac{\sum_{n=0}^{\frac{N}{2}-1}\left(I_{\|}(n)-I_{\perp}(n)\right)}{\sum_{n=0}^{\frac{N}{2}-1}\left(I_{||}(n)+I_{\perp}(n)\right)}
$$

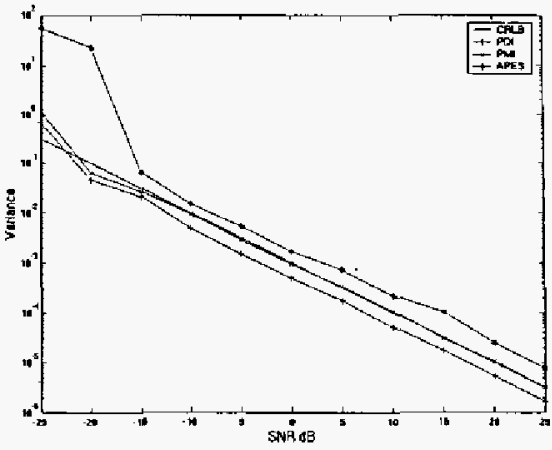

Fig. 3. Variance of estimators of DOLP in white noise.

and

$$
\widehat{D O L} P_{P D I 2}=\frac{2}{N} \sum_{n=0}^{\frac{N}{2}-1}\left(\frac{I_{\|}(n)-I_{L}(n)}{I_{\|}(n)+I_{\perp}(n)}\right)
$$

It is clear that all the estimators derived from $(4.0 .1)$ will be biased, since the numerator corresponds to scaled versions of the polar ization intensity data, which was shown to be biased. However, if $\beta=\frac{\pi}{2}$ in (3) and (4), or if the relative (rather than the actual) values of DOLP in a scene are of interest, then, $\overparen{D O L} P_{P D I}$ can be used. However, if $\beta \rightarrow 0$, the noise may completely obscure the DOLP information. Henceforth, we assume $\beta=\frac{\pi}{2}$, so that the numerator is an unbiased estimate of $I_{b a l}$.

The form of $\overline{D O L} P_{P D I}$ is that of a ratio estimator, with both the numerator and the denominator being Gaussian random variables which are uncorrelated in case the noise is white, but correlated in case of coloured noise. A comparative study of these estimators has been reported in [13]. It has been found that $\overparen{D O L P} P_{P D 2}$ estimator is biased and $\overparen{D O L} P_{P D I}$ is asymptotically unbiased. The variances of the estimators are equal and diminish to zero asymptotically. Hence, both the estimators are consistent. For these reasons, it is better to use $\widehat{D O L} P_{P D I}$. It can also be observed that the memory requirements of $D \widehat{O L} P_{P D I 1}$ is less than that of $\overrightarrow{D O L} P_{P D I 2}$.

\subsubsection{The PMI estimator of DOLP}

In our analysis of the PII, we found that, for $\beta \neq \frac{\pi}{2}$, we can obtain only MLE of $I_{\text {brat }}$ and not its MVU estimate. We can show that the MLE of $I_{\text {scat }}$ also corresponds to its MVU estimate in both coloured and white noise. Hence, we can obtain MLE of DOLP using the invariance property of the MLE [11]. The transformation $\widehat{D O L} P=\frac{i_{b u l}}{\hat{I}_{\text {acat }}}$ being non-invertible, the MLE maximizes a modiffed likelihood function, as explained in [11]. By the theorem on the asymptotic behaviour of the MLE [11], we conclude that the MLE achieves the CRLB asymptotically.

\subsection{Results from Monte-Carlo simulations}

Figs. 3, 4 show the performance of the various estimators in white and ARl noise, respectively. We can observe that PDI and PMI estimators seem to be the best for estimating DOLP too, with the PDI performing better than the PMI (with $\beta=\pi / 2$ ). Though MLE and APES estimators also perform as good as PMI estimator, their 


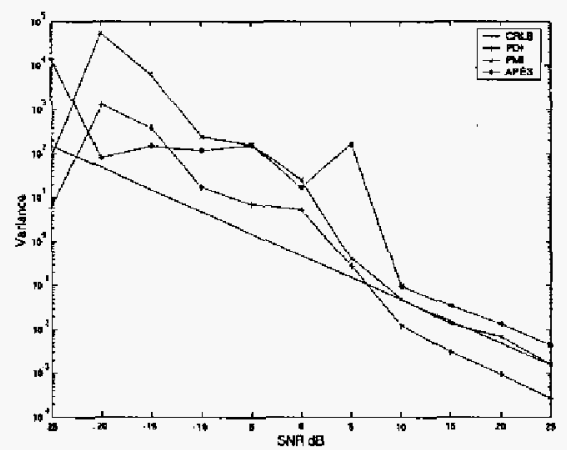

Fig. 4. Variance of estimators of DOLP in AR1 noise.

computational costs are much higher. Hence, PDI or PMI should be preferred over them.

\section{EXPERIMENTAL RESULTS}

Experiments were conducted to obtain shadowgrams of an opaque object immersed in calibrated solutions containing polystyrene microspheres dispersed in water. We observed that with microsphere of diameter $2.97 \mu$, we could inage up to an optical thickness of around 6.77 , while we could image up to an optical thickness of 40 in samples containing polystyrene beads of diameter $0.11 \mu$. A result obtained from one of the data sets is shown in Fig.5. Fig 5(a) shows the shadow of the object without scattering. Fig 5(b) shows a representative image in the scries of images acquired. Figs 5 (c), 5 (d) and 5(e) show the polarization intensity imaging result obtained by processing 16 images recorded using the PDI, PMI and the APES schemes, respectively. The experimental setup used, was similar to the one given in [4]. $\beta$ was nearly $\pi / 2$ in the experiment reported here. The superiority of the PDI scheme can be easily observed by comparing the results, thereby endorsing our theoretical observations.

\section{REFERENCES}

[1] Jeremy C Hebden, Simon R Arridge, and David T Delpy, "Optical imaging in medicine: 1 . experimental techniques," Phys.Med.Biol, vol, 42, pp. 825-840, 1997.

[2] M P Rowe, E N PughJr, J S Tyo, and N Engheta, "Polarization-difference imaging: a biologically inspired technique for observation through scattering mediu," Optics Letters, vol. 20, no. 6, pp. 608-610, Mar. 1995.

[3] J S Tyo, M P Rowe, E N PughJr, and N Engheta, "Target detection in optically scattering media by polarizationdifference imaging;" Applied Optics, vol. 35, no. 11, pp. 1855-1870, Apr. 1996.

[4] Ramachandran Hema and Narayanan Andal, "Twodimensional imaging through turbid media using a continuous wave light source," Optics Communications, vol. 154, pp. 255-260, Sept. 1998.

[5] Oliver Emile, Fabien Bretenaker, and Albert Le Floch, "Rotating polarization imaging in turbid media," Optics Letters, vol. 21 , no. 20, pp. 1706-1708, Oct. 1996.

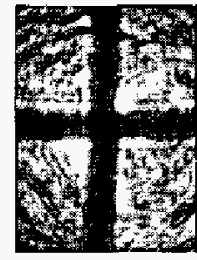

(a)

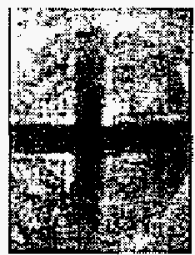

(c)

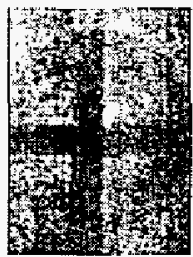

(e)

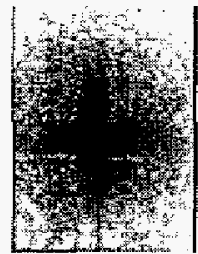

(b)

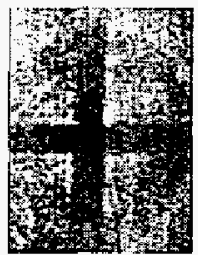

(d)

(a) Actual object.

(b) Unprocessed image.

(c) PDI result.

(d) PMI result.

(e) APES result.
Fig. 5. Experimental results.

[6] Craig F Bohren and Donald R Huffman, Absorption and Scattering of Light by Small Particles, John Wiley and Sons Inc, New York, 1998.

[7] William S Bickel and Wilbur M Bailey, "Stokes vectors, mueller matrices, and polarized scattered light," American Journal of Physics, vol. 53, no. 5, May 1985.

[8] Serge Huard, Polarization of light, John Wiley \& Sons, 1997.

[9] Christian Brosseau, Fundamentals of polarized light-A siatistical optics approach, John Wiley and Sons Inc, New York, 1998.

[10] John G Walker, Peter C Y Chang, and Keith I Hopcraft, "Visibility depth improvement in active polarization imaging in scattering media," Applied Optics, vol. 39, no. 27, pp. 49934941, Sept. 2000.

[11] Steven M kay, Fundamentals of Statistical Signal Processing: Estimation Theory, Prentice Hall International Inc., New Jersey, 1993.

[12] Petre Stoica, Hongbin Li, and Jian Li, "Amplitude estimation of sinusoidal signals: Survey, new results, and an application," IEEE Trans. on signal processing, vol. 48, no. 2, pp. 338-352, Feb. 2000.

[13] G M P van Kempen and L J van Vliet, "Mean and variance of ratio estimators used in fluorescence ratio imaging," $C y-$ tometry, vol. 39, pp. 300-305, 2000. 